\title{
A Methodology to Improve the Speed of Communication in Automobile
}

\author{
Shubhangi Dagwar \\ India
}

\begin{abstract}
In recent years there has been a significant increase in the amount of electronics that have been introduced into the car, and this trend is expected to continue as car manufacturers introduce further advances in safety, reliability and comfort. The introduction of advanced control systems combining multiple sensors, actuators and electronic control units are beginning to place demands on the communication technology that were not previously addressed by existing communication protocols.

Additional requirements for future in-car control applications include the combination of higher data rates, deterministic behavior and the support of fault tolerance.

Flexibility in both bandwidth and system extension will also be key attributes as the need for increased functionality and on-board diagnostics also increase.

Availability, reliability and data bandwidth are the key for targeted applications in Powertrain, Chassis and Body control, and these must also be supported within the automotive environment which presents some unique challenges, in this paper so we are introducing flexray protocol for next generation car.
\end{abstract}

The full text of the article is not available in the cache. Kindly refer the IJCA digital library at www.ijcaonline.org for the complete article. In case, you face problems while downloading the full-text, please send a mail to editor at editor@ijcaonline.org 